\title{
Physicochemical Characterization and Cell Adhesion Evaluation of Poly-E-Caprolactone/BNNT Nanofibers Produced through Electrospinning Process
}

\author{
Tiago H. Ferreira ${ }^{1}$, Anderson Maia Peres ${ }^{1,2}$, Jerusa A.Q.A. Faria ${ }^{3}$, Dawidson Assis Gomes ${ }^{3}$, \\ Tadeu H. Lima ${ }^{2}$, Rodrigo Lambert Oréfice ${ }^{2}$, Edésia Martins B. Sousa ${ }^{*}$ \\ ${ }^{1}$ Centro de Desenvolvimento da Tecnologia Nuclear-CDTN, 31270-901 Belo Horizonte, MG, Brazil \\ ${ }^{2}$ LEPCom - Departamento de Engenharia Metalúrgica e de Materiais - ICEX - UFMG - Belo Horizonte - MG \\ - Brazil \\ ${ }^{3}$ Departamento de Bioquímica e Imunologia - ICB - UFMG - Belo Horizonte - MG - Brazil
}

*Corresponding Author: Edésia Martins B. Sousa, Centro de Desenvolvimento da Tecnologia Nuclear - CDTN, 31270-901 Belo Horizonte, MG, Brazil

\begin{abstract}
Hybrid nanostructured materials have been broadly studied in the last years. Boron nitride nanotubes (BNNTs) have chemical and thermal stability, in addition to its excellent mechanical properties. Furthermore, the fluorescence presented by these BNNTs can be useful for many applications. On the other hand, Poly-E-caprolactone (PCL) is a widely used polymer in biomedical and pharmaceutical applications. PCL is biocompatible and may be degraded by hydrolytic and enzymatic reactions without toxicity. Hence, PCL nanofibers, produced by electrospinning, can be applied as scaffolds for tissue engineering. In this study, boron nitride nanotubes (BNNTs) were incorporated in the matrix of PCL nanofibers through electrospinning process. This hybrid nanomaterial was systematic characterized through thermogravimetry analysis, Fourier transformation infrared spectroscopy (FTIR) and the morphological characteristics were available by scanning electron microscopy (SEM) and transmission electron microscopy (TEM). Furthermore, the biological experiments showed that the hybrid nanomaterial is biocompatible, and allows cell adhesion. From the obtained results it was possible to confirm that this hybrid system has the desired features for application as biosensor and tissue engineering.
\end{abstract}

Keywords: Cell adhesion, Poly-ع-caprolactone, nanofibers, BNNT and Fluorescence.

\section{INTRODUCTION}

The advances in the field of nanomaterials have achieved a high level of sophistication that made it acquire a more important role in solving the current problems of science. Thereby, with every passing day, the nano-sciences have contributed more effectively to scientific and technological developments. The use of distinct components in which each part contributes in a different manner to perform multiple functions in a single material has been an integral part of this advanced materials approach. The improvement in synthesis processes allows the development of complex systems of various shapes and sizes with proper control of composition, porosity, functionality, and morphology. The huge abundance of these hybrid structures and the possibility to combine different properties of each one assure the continued progress that will make it possible to overcome economic and technical limitations in current technology [1].

Polymeric nanofibers produced by electrospinning process have been explored recently mainly due to the mechanical biological and kinetic properties of the scaffold being easily manipulated by altering the polymer composition and processing parameters [2]. Nanofiber scaffolds serve as suitable environment for cell attachment and proliferation due to similarity and compatible to physical dimension of natural extracellular matrix [3].

The high surface area and pore density make the electrospun nanofibers suitable to allow cell adhesion, growth and differentiation of cells [4,5]. For all these characteristics, the nanofibers emerged as a promising tool such as supports for culture of cells and tissues [6], vascular grafts [4], 
extracellular matrices [7], carriers for topical/transdermal drug delivery [8], electrical and optical applications $[9,10]$, sensors and high performance structures $[11,12]$.

Optoelectronic devices are usually fabricated with the incorporation of optoelectronically active materials such as conjugated polymers, quantum dots and/or fluorescence nanoparticles into electrospun nanofibers. A fluorescent composite nanofiber can be obtained with the addition of a light emitter nanoparticle into an inert polymeric matrix. PCL is a semicrystalline aliphatic polyester with biodegradable and biocompatible properties approved of the Food and Drug Administration for many biomedical applications. PCL is one of the most commonly used biomaterials for fabrication via electrospinning of nanofibers with submicron diameters [13]. Furthermore, in addition to intrinsic properties, PCL nanofibers (nPCL) can serve as support for fluorescence applications [14].

An innovative class of nanomaterials is represented by nanotubes, among them: carbon nanotubes (CNTs) [15], titanate nanotubes (TNTs) [16] and boron nitride nanotubes (BNNTs) [17]. BNNT is a structural analogue of CNT formed with tubular shaped hexagonal boron nitride (hBN) sheet in which $\mathrm{C}$ atoms are substituted by alternating $\mathrm{B}$ and $\mathrm{N}$ atoms in a resonance hybrid that presents a high thermal stability and good chemical inertia. Furthermore, these hybrids contain interesting mechanical and electrical properties that could be useful for many applications. Thanks to their special chemical and physical characteristics, they have already found a large number of applications in the field of nanotechnology. Some studies concerning the interactions between nanotubes and biological systems have been mainly focused on biomedical applications, including their use as biosensors [18], nanovectors [19] and many others [20]. In order to form a hybrid system that includes the characteristics of both materials, it was proposed in this work the addition of BNNTs into a nPCL matrix. Taking advantage of the detectable signal through fluorescence microscope coming from BNNTs [21], it may be possible to modulate their emission in response to the adsorption of specific biomolecules, as already shown for carbon nanotubes [22] [23]. Thereby, it can be possible to develop a biosensor based on this hybrid system. The goal of this study was to investigate the physicochemical characterization and behavior of electrospun PCL and BNNT nanofiber scaffold for future tissue engineering strategies.

\section{Materials AND Methods}

Amorphous boron powder, ammonium nitrate, hematite, Poly- $\varepsilon$-caprolactone (80000 MW), acetic acid and formic acid were obtained from Sigma-Aldrich (São Paulo-Brazil). All solvents used in this study were of analytical grade. All other chemicals used in this study were available commercially as a reagent grade and were used without further purification. MilliQ ${ }^{\circledR}$ water (simplicity 185, Millipore, Bedford, USA) was used throughout the study.

\subsection{Synthesis of BNNTs}

BNNTs samples were prepared as previously described [24]. The powders of $\mathrm{NH}_{4} \mathrm{NO}_{3}(95 \%$, w/w), amorphous boron $(97 \%, \mathrm{w} / \mathrm{w})$, and hematite $(95 \%, \mathrm{w} / \mathrm{w}$ and particle size less than $50 \mathrm{~nm})$ were mixed at a molar ratio of 15:15:1, respectively, placed in tubular furnace and they were subjected to a heat treatment. After, the BNNTs were purified with hydrochloric acid solution $(3 \mathrm{M})$ at $90^{\circ} \mathrm{C}$ for 10 minutes, and then the sample was collected by filtration and dried at $40^{\circ} \mathrm{C}$.

\subsection{Preparation of PCL/BNNTs nanofibers}

The nanofibers were prepared with BNNTs in a ratio of $3 \%$ by weight of the polycaprolactone. This concentration was chosen after preliminary tests that defined the best proportion to make the nanofiber with appropriate brightness and uniformity.

The BNNTs were added to a solution of acetic acid/formic acid (1:1) and sonicated for 3 minutes $(3000 \mathrm{KJ}$ of energy). After this, the polymer was slowly added $(15 \% \mathrm{w} / \mathrm{v})$ in the dispersion, under magnetic stirring at room temperature, and these conditions were kept constant for $2 \mathrm{~h}$. For electrospinning process, the collector was positioned to $8 \mathrm{~cm}$ away from the needle to the deposition of the nanofibers. The dispersion was injected through a $23 \mathrm{G}$ blunt tip syringe at a rate of $3.5 \mathrm{ml} / \mathrm{h}$, and a high voltage was applied, $18 \mathrm{kV}$ in the needle tip for approximately $90 \mathrm{~min}$. The representative scheme for obtaining of nPCL/BNNT is shown in Figure 1. The hybrid material was dried at room temperature for 1 day, and then was stored in a desiccator before testing. 


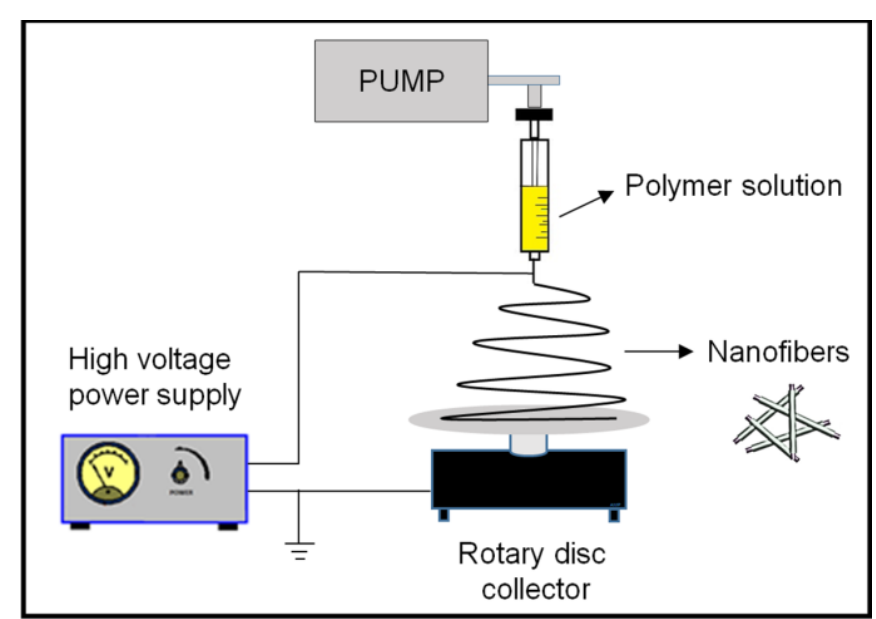

Figure1. Representative scheme for obtaining of $n P C L / B N N T$

\subsection{Physicochemical Characterization}

2.3.1. The presence and percentage of the BNNTs on the $\mathrm{nPCL}$ were evaluated by thermogravimetric analysis (TGA). TGA measurements were taken by EXSTAR TG/7200 at a temperature ranging from 25 to $800{ }^{\circ} \mathrm{C}$. Approximately $5.0 \mathrm{mg}$ of sample was analyzed with a heating rate of $20^{\circ} \mathrm{C} \cdot \mathrm{min}^{-1}$, using a nitrogen atmosphere flow of $30 \mathrm{ml} / \mathrm{min}^{-1}$ and an open alumina pan.

2.3.2. Fourier transform infrared spectroscopy (FTIR). The FTIR measurements were conducted to complement the results obtained from thermal analysis experiments. The procedure was conducted in a PerkinElmer 1760-X spectrophotometer in the range from 4000 to $650 \mathrm{~cm}^{-1}$ with 64 scans and $4 \mathrm{~cm}^{-1}$ of resolution.

2.3.3. X-ray diffraction (XRD). The crystalline phases of samples were examined with X-ray powder diffraction. The XRD patterns were obtained using a Rigaku Geigerflex-3034 diffractometer with a $\mathrm{Cu}-\mathrm{K} \alpha$ tube.

2.3.4. Scanning Electron Microscopy - SEM. The morphological characteristics of BNNTs, nPCL and nPCL/BNNTs were studied using the JEOL JSM, 840A operating at $15 \mathrm{kV}$. The samples were placed on a carbon tape and metallized with gold (SPI Suplies - Sputter coater). The images were obtained in electron secondary mode, where a minimum of 10 images from each sample were analyzed to assure reproducible results.

2.3.5. Transmission electron microscopy (TEM). The morphological characteristics of BNNTs, nPCL and nPCL/BNNTs were studied using the Tecnai G2-20-FEI 2006 with an acceleration potential of $200 \mathrm{kV}$. The nanofibers were deposited directly on the transmission grid during the electrospinning process.

\subsection{Biological Assay}

\subsubsection{Cell Culture}

The MRC-5, a human lung fibroblast cell line (ATCC, VA, USA) was cultured at $37^{\circ} \mathrm{C}$ in $5 \% \mathrm{CO} 2$ in Dulbecco's modified Eagle's medium (Life Technologies, NY, USA) containing $10 \%$ fetal bovine serum (FBS), $1 \mathrm{mM}$ sodium pyruvate, 50 units/mL penicillin, and $100 \mu \mathrm{g} / \mathrm{mL}$ streptomycin (Life Technologies).

\subsubsection{Cell Viability Assay of Electrospun Nanofibers}

The WST-8 assay was used to evaluate cell viability, which are based on the conversion of a watersoluble tetrazolium salt, 2-(2-methoxy-4-nitrophenyl)-3-(4-nitrophenyl)-5-(2,4-disulfophenyl)-2Htetrazolium, monosodium salt, to a water-soluble formazan dye upon reduction by dehydrogenases in the presence of an electron carrier [25]. Electrospun nanofiber were prepared on circular coverslips (13 $\mathrm{mm}$ in diameters) and sterilized by UV irradiation. $1 \times 10^{5}$ cells were plated on nanofibers and glass coverslips, which were used as controls. After 24 and $48 \mathrm{~h}$, the extent of cell viability was assessed using a Cell Counting Kit - 8 (CCK-8 - Sigma-Aldrich). The CCK-8 solution (10 $\mu \mathrm{L})$ was added to each well, followed by incubation for $2 \mathrm{~h}$ at $37^{\circ} \mathrm{C}$. The absorbance at $450 \mathrm{~nm}$ was determined using a microplate reader (Multiskan GO; Thermo Scientific). Cell viability was expressed as a percentage relative to the untreated cells (control). The experiments were carried out in 
triplicate. Significance of changes in treatment groups was determined by one-way analysis of variance and Bonferroni's multiple comparison tests, using Prism 6 software. Data were represented as mean \pm S.E.M.

\subsubsection{Live Cell Imaging Using Confocal Microscopy}

Confocal microscopy was used to evaluate the cells morphology and viability cultivated on nanofibers. 1 x $10^{5}$ MRC-5 cells were plated in 24-well tissue culture plate with nPCL and BNNT nanofibers coverslips. After 48 hours, cells were stained with $1 \mu \mathrm{M}$ of Calcein Acetoxymethyl Ester (Calcein-AM) dissolved in DMEM without FBS and incubated at $37^{\circ} \mathrm{C}$ for 30 minutes in a $5 \% \mathrm{CO}_{2}$ incubator. After staining, the cells were washed twice with 1x PBS. The images were collected using a Zeiss LSM 880 with the super-resolution Airyscanner using a 20X/0.8 NA objectives lens. CalceinAM and BNNT fluorescence were excited with $488 \mathrm{~nm}$ and $633 \mathrm{~nm}$, respectively.

\section{RESULTS}

Initially, BNNT nanotubes, nPCL and the hybrid nPCL/BNNTs were investigated for the physicochemical characterization.

The weight losses from the BNNT, nPCL and nPCL/BNNTs systems were evaluated in the range from 25 to $800{ }^{\circ} \mathrm{C}$. The TGA thermograms of all samples are shown in Figure 2a. BNNTs exhibit thermal stability throughout the heating interval and no significant weight loss could be identified. PCL nanofibers have a thermal decomposition profile between $320-500{ }^{\circ} \mathrm{C}$. At $430{ }^{\circ} \mathrm{C}, 50 \%$ of nanofibers were thermally degraded. From $650{ }^{\circ} \mathrm{C}$, the nPCL were completely decomposed and no trace of residue was presented. In the cases of nPCL/BNNTs sample, weight loss occurred in the same region of PCL nanofibres, between $320-500{ }^{\circ} \mathrm{C}$. The incorporation of the BNNTs into nPCL is confirmed throught of residue about $3 \%$, after $650{ }^{\circ} \mathrm{C}$, related to the BNNTs. This behavior can be better viewed in Figures $2 \mathrm{~b}$ by analyzing the derivates of thermogravimetric curves (DTG) for the BNNT, nPCL and nPCL/BNNTs samples.
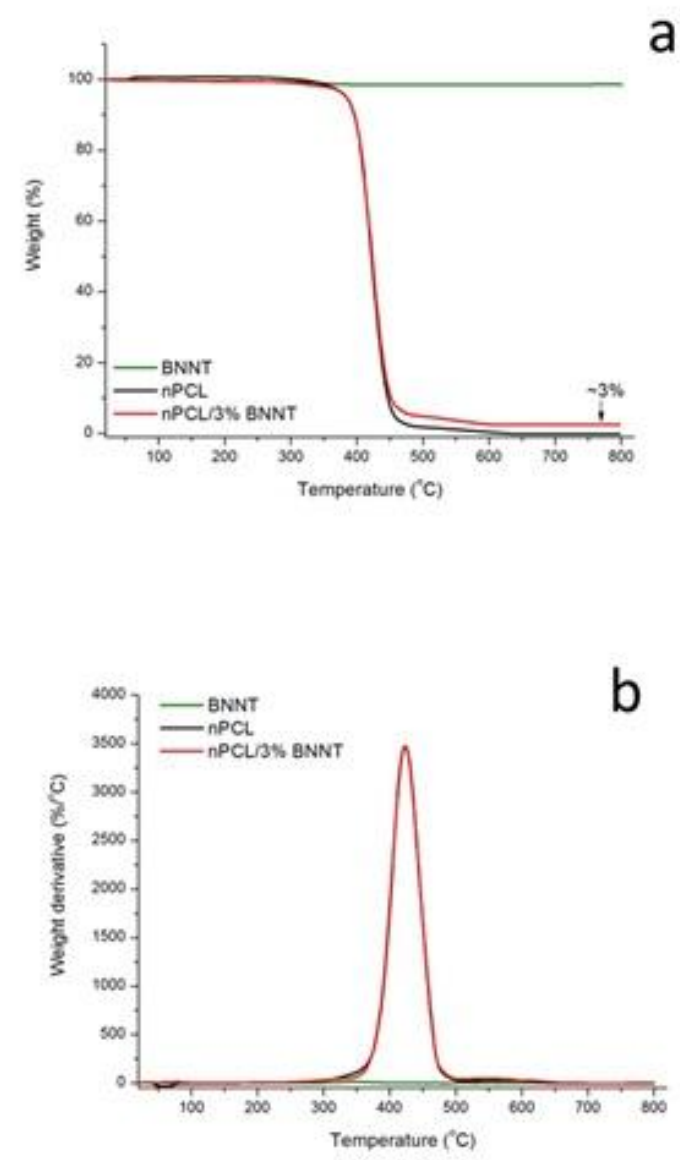

Figure2. Thermogravimetric analysis of BNNT; nPCL and nPCL / 3\% BNNT. (a) TGA and (b) DTG 
Figure 3a shows a comparative FTIR spectrum study of BNNTs, nPCL and nPCL/BNNTs. BNNTs have characteristics bands which are a strong asymmetric band centered at $1380 \mathrm{~cm}^{-1}$, which corresponds to the bond B-N stretch, along with a less intense band at $790 \mathrm{~cm}^{-1}$ attributed to B-N-B bond [26]. The main absorption bands in the infrared for $\mathrm{nPCL}$ are $2949 \mathrm{~cm}^{-1}$ and $2865 \mathrm{~cm}^{-1}$ associated with asymmetrical and symmetrical stretching of $\mathrm{CH}_{2}$, respectively, $1727 \mathrm{~cm}^{-1}$ associated with the stretching of the carbonyl, $1293 \mathrm{~cm}^{-1}$ to CO and CC stretching in the crystalline phase, 1240 $\mathrm{cm}^{-1}$ associated with the COC asymmetric stretching and $1170 \mathrm{~cm}^{-1}$ associated with the COC symmetrical stretching $[27,28]$. The presence of the band at $790 \mathrm{~cm}^{-1}$ in the nPCL/BNNTs indicates again the presence of BNNT in the nPCL.

The crystalline structure of the samples was investigated by XRD; the profile is shown in Figure $3 \mathrm{~b}$. PCL is a semicrystalline polymer with two diffraction peaks at $2 \theta=21.4^{\circ}$ and $23.6^{\circ}$, which correspond to the orthorhombic planes (110 and 200) [29]. The XRD pattern of the resulting nPCL presents these two characteristics peaks, and also in the sample nPCL/BNNTs. The peaks at $2 \theta=$ $26.7^{\circ}$ and $41.6^{\circ}$ (JCPDS, No. 9-12) correspond to the diffraction planes (002 and 102) of hexagonal boron nitride that are related to the formation of BNNTs. These peaks are clearly visualized in the BNNTs sample, however in nPCL/BNNTs it is only possible to observe the main peak at $2 \theta=26.7^{\circ}$.

Just as occurs with the measurements of FTIR, in XRD the characteristic signals of PCL are readily observed in the hybrid system. However, due the smaller proportion of BNNTs contained in the polymer matrix, its signal appears with restrained intensity.
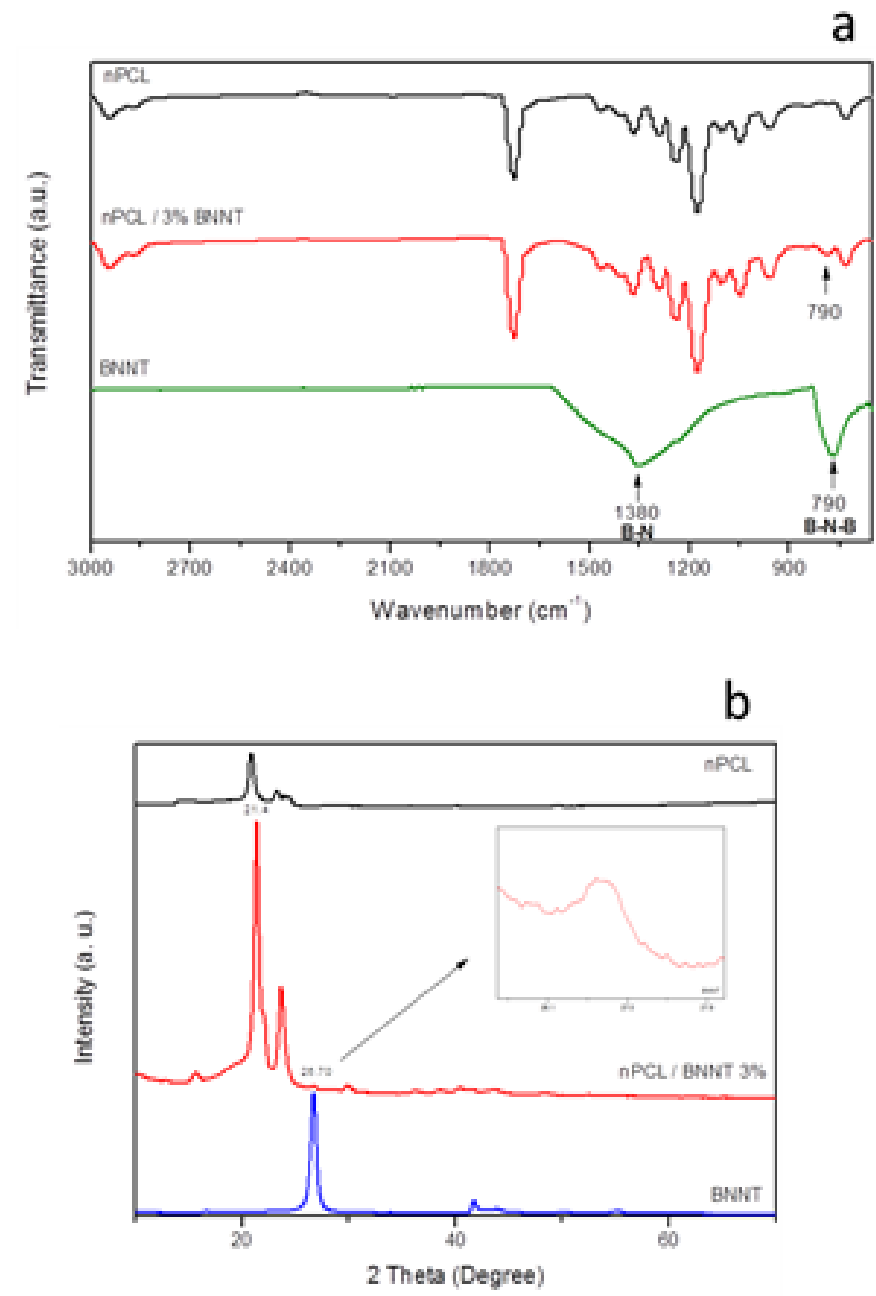

Figure3. FTIR (a) and XRD (b) of BNNT; nPCL and nPCL/3\% BNNT samples

The SEM images of the samples are shown in Figure 4. Nanotubes obtained through this synthesis route have diameter of about $100 \mathrm{~nm}$. The presence of some nanoparticles of BN also can be observed in Figure 4a. Figure $4 \mathrm{~b}$ shows the morphology of nPCL and demonstrate that the nanofibers produced 
were smooth, uniform, bead-free and with a diameter of approximately $230 \mathrm{~nm}$. In the hybrid nPCL/BNNTs (Figure 4c), the nanofibers maintain its morphology however the average diameter was increased to about $340 \mathrm{~nm}$. This happens because the dispersion of BNNT in PCL may have increased viscosity, thereby increasing the nanofiber diameter. The increase in diameter of electrospinning nanofibers is expected with increase the polymer solution viscosity [30].
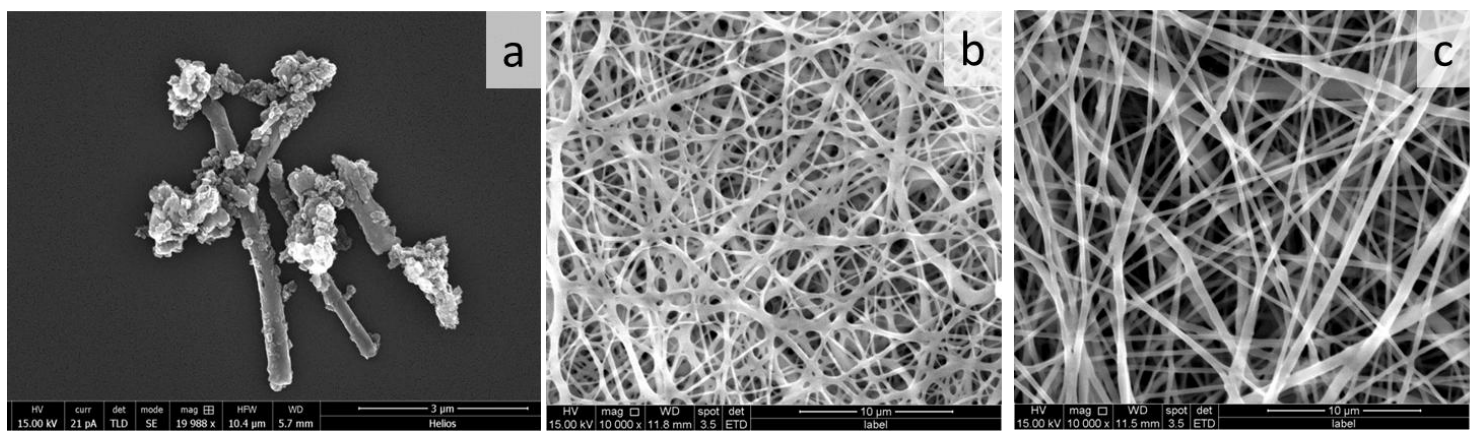

Figure4. SEM images of BNNT (a); $n P C L(b)$ and $n P C L / 3 \%$ BNNT

In Figure 5a the TEM image of BNNTs evidence the multiwalled nature of the BNNTs having an outer diameter of approximately $70 \mathrm{~nm}$. The layers spaced by $0.33 \mathrm{~nm}$, corresponds to the crystallographic plane 002 [31].

The $n$ PCL/BNNTs can be observed in Figure $5 b$ in bright field. Even after addition of nanotubes the standard morphology of nanofibers is kept and the average diameter is in accordance with SEM images. Diffraction contrast in TEM investigations is derived from scattering by crystalline regions oriented in the Bragg condition with respect to the incident electron beam [32]. The materials with crystalline regions that can produce strong Bragg reflections falling into the angular selection range of the objective aperture in TEM show bright contrast in dark-field mode provided the crystalline orientation of these materials and contributed to image formation. Therefore the dark-field image (Figure 5c) was obtained from the scattered signal relating to plane [002] of BNNT. Thus, the nPCL can be observed as dark spots and BNNT as bright spots dispersed on the nanofibers matrix. We can observe in this image that BNNTs were not well distributed in all nanofibers.
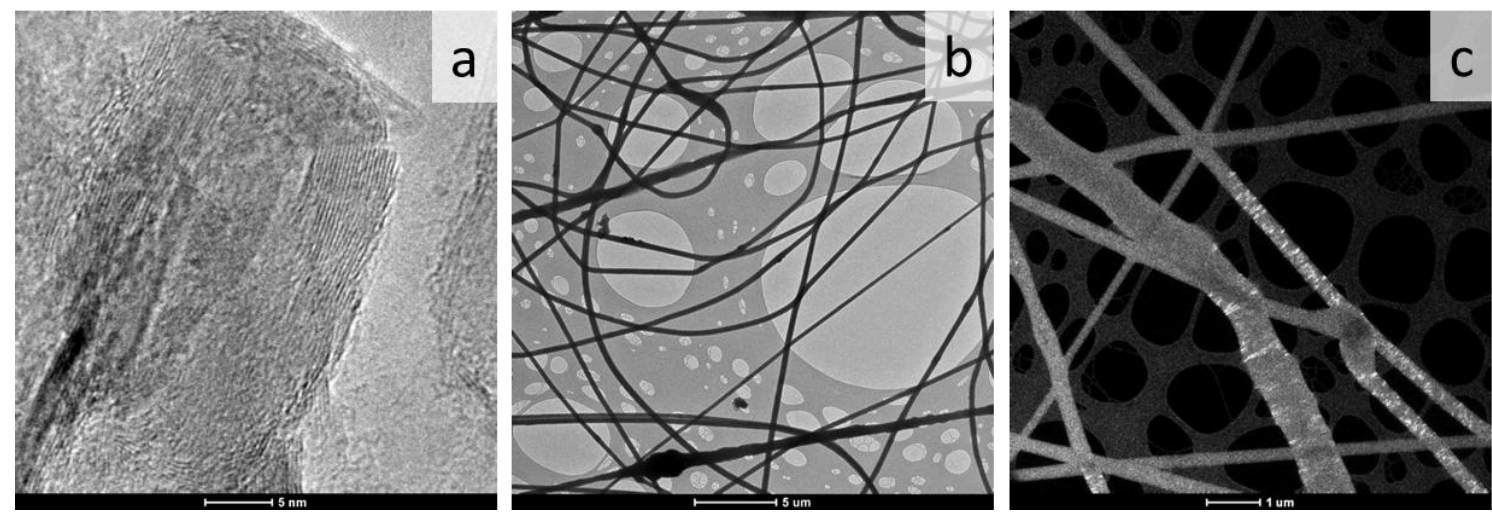

Figure5. TEM images of BNNT (a); $n P C L / 3 \%$ BNNT in bright field $(b)$ and in dark field (c)

Cell viability assay employing Calcein-AM staining was performed to ensure the viability and assess the morphology of MRC-5 cells on nanofibers scaffolds. The membrane-permeable Calcein-AM in live cells is converted to a green-fluorescent calcein after acetoxymethyl ester hydrolysis by intracellular esterases. Confocal images showed that the cells cultured for 48 hours were capable to adhere on nPCL/BNNTs scaffolds with its characteristic fibroblastoid morphology (Figure 6a). Corroborating this data, the three-dimensional reconstruction of serial confocal immunofluorescence showed that viable cells were localized in the inner region of BNNT nanofiber. Also, the BNNT fluorescence was observed using a long pass $660 \mathrm{~nm}$ filter with the excitation at $647 \mathrm{~nm}$ (Figure 6b, $3 \mathrm{D}$ reconstruction of Z-stack images in supplementary video 1 ).

Scaffolds containing nanofibers cell viability did not show significant difference compared to control after 24 hours of plating. However, nPCL/BNNTs showed significantly reduced cell viability related 
to control after 48 hours (Figure 6c). Collectively, cell viability assay and confocal images indicated that the polymer surface was highly biocompatible, as fibroblast cells could favorably attach on the polymer surface.

A
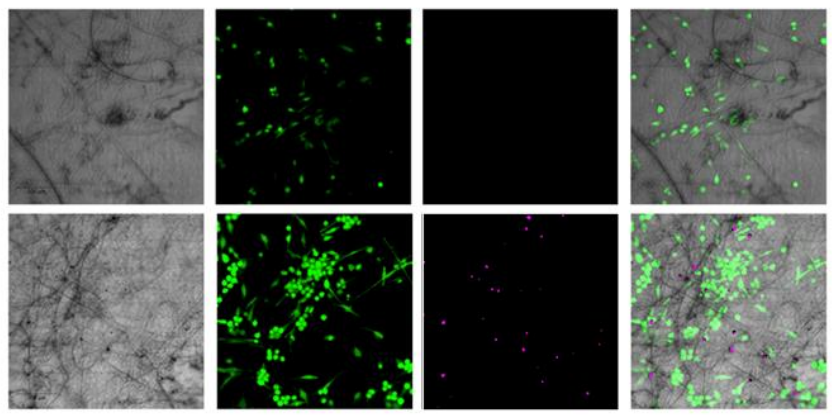

B

C
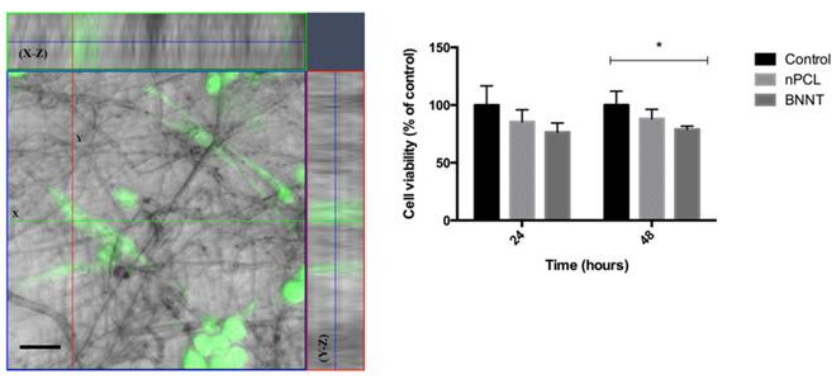

Figure 6 - Cell cultured on nanofibers retains cell viability and cell morphology. A. Representative fluorescence images of live cells in nanofibers scaffolds. MRC-5 cells were stained with calcein-AM (green) and magenta (BNNT). Scale bar $=100 \mu \mathrm{m}$. B. Serial optical sections were collected for threedimensional reconstruction; $\mathrm{x}-\mathrm{z}$ sections are shown at the top, and $\mathrm{y}-\mathrm{z}$ sections are shown at the right of each image. Bar $=20$ micrometers. C. Cell viability assay on the electrospun scaffolds after 24 and 48 hours of cell seeding. Cells were incubated with CCK-8 solution for 2 hours and ability of attached cells to reduce WST-8 was calculated. *, p $<0.05$ BNNT compared to control group.

\section{DISCUSSION}

Scaffold nPCL nanofibrous is a promising candidate for tissue engineering applications [33,34]. In this work, the nanofibers scaffolds were cultured with MRC-5 cells to investigate whether nanofibers PCL or BNNT/PCL have biocompatibility to the cells. The confocal microscopy images and WST-8 assay showed that cells is able to migrate on the nanofibers and these biomaterial supports cell adhesion and viability. Many researches have been shown that cells can penetrate into the small porous of PCL nanofiber [34,35]. Corroborating with that, three-dimensional reconstruction of serial confocal images confirmed that living cells migrate throughout the nPCL/BNNTs.

The different functionalization process using electron-withdrawing molecules are shown to provide sites for transferring electrons in and out of the nanotube. In some cases the fluorescence energy of the nanotube can be sensitive enough to be detected through an energy shift that is distinct and measurable from an arbitrary sequence [36]. These mechanisms involved in the functioning of a sensor must be extensively studied in our specific system so that the proposed application can be achieved.

\section{CONCLUSION}

Through experiments conducted in the present study, the BNNTs were successfully incorporated into matrix of PCL nanofibers through electrospinning process. The morphology of nanofibers was maintained, despite there was a small increase in the average diameter. The fluorescence of BNNT, which was kept in the hybrid, confirms that the nanoparticles present an adequate dispersion. The scaffold formed by nPCL/BNNT is biocompatible, and allows cell adhesion. Several important points 
need to be further studied and improved, however the collected evidences are encouraging, and suggest that hybrid systems based on BNNT are suitable to be used as biosensors and tissue engineering.

\section{ACKNOWLEDGEMENTS}

The authors thank for FAPEMIG (Fundação de Amparo a Pesquisa do Estado de Minas Gerais), CNPQ (Conselho Nacional de Desenvolvimento Científico e Tecnológico), and CAPES (Comissão Aperfeiçoamento de Pessoal de Nível Superior) for their financial support, as well as the CEMUFMG (Centro de Microscopia da UFMG) and CAPI-ICB/UFMG (Centro de Aquisição e Processamento de Imagens) for microscopy images.

\section{REFERENCES}

[1] C. Sanchez, K.J. Shea, S. Kitagawa, Recent progress in hybrid materials science., Chem. Soc. Rev. 40 (2011) 471-472.

[2] E.J. Chong, T.T. Phan, I.J. Lim, Y.Z. Zhang, B.H. Bay, S. Ramakrishna, et al., Evaluation of electrospun PCL/gelatin nanofibrous scaffold for wound healing and layered dermal reconstitution., Acta Biomater. 3 (2007) 321-30.

[3] Z. Ma, M. Kotaki, R. Inai, S. Ramakrishna, Potential of nanofiber matrix as tissue-engineering scaffolds., Tissue Eng. 11 101-9.

[4] H. Wang, Y. Feng, Z. Fang, R. Xiao, W. Yuan, M. Khan, Fabrication and characterization of electrospun gelatin-heparin nanofibers as vascular tissue engineering, Macromol. Res. 21 (2013) 860-869.

[5] D. Yang, Y. Li, J. Nie, Preparation of gelatin/PVA nanofibers and their potential application in controlled release of drugs, Carbohydr. Polym. 69 (2007) 538-543.

[6] E. Kijeńska, M.P. Prabhakaran, W. Swieszkowski, K.J. Kurzydlowski, S. Ramakrishna, Interaction of Schwann cells with laminin encapsulated PLCL core-shell nanofibers for nerve tissue engineering, Eur. Polym. J. 50 (2014) 30-38.

[7] P. Kuppan, S. Sethuraman, U.M. Krishnan, PCL and PCL-gelatin nanofibers as esophageal tissue scaffolds: Optimization, characterization and cell-matrix interactions, J. Biomed. Nanotechnol. 9 (2013) $1540-1555$.

[8] G. Kim, H. Yoon, Y. Park, Drug release from various thicknesses of layered mats consisting of electrospun polycaprolactone and polyethylene oxide micro/nanofibers, Appl. Phys. A Mater. Sci. Process. 100 (2010) 1197-1204.

[9] R. Sengupta, S. Chakraborty, S. Bandyopadhyay, S. Dasgupta, R. Mukhopadhyay, K. Auddy, et al., A Short Review on Rubber / Clay Nanocomposites With Emphasis on Mechanical Properties, Engineering. 47 (2007) 21-25.

[10] S. Sheng, Q. Ma, X. Dong, N. Lv, J. Wang, W. Yu, et al., Single flexible nanofiber to achieve simultaneous photoluminescence-electrical conductivity bifunctionality, Luminescence. 30 (2015) 26-31.

[11] J. Wei, S. Yang, L. Wang, C.-F. Wang, L. Chen, S. Chen, Electrospun fluorescein-embedded nanofibers towards fingerprint recognition and luminescent patterns, RSC Adv. 3 (2013) 19403.

[12] L.-N. Chen, C.-C. Kuo, Y.-C. Chiu, W.-C. Chen, Ultra metal ions and pH sensing characteristics of thermoresponsive luminescent electrospun nanofibers prepared from poly(HPBO-co-NIPAAm-co-SA), RSC Adv. 4 (2014) 45345-45353.

[13] S. Hong, G. Kim, Fabrication of size-controlled three-dimensional structures consisting of electrohydrodynamically produced polycaprolactone micro/nanofibers, Appl. Phys. A Mater. Sci. Process. 103 (2011) 1009-1014.

[14] R. Bagherzadeh, M. Latifi, S.S. Najar, M. a. Tehran, L. Kong, The application of Cd Se/ZnS quantum dots and confocal laser scanning microscopy for three-dimensional imaging of nanofibrous structures, J. Ind. Text. (2012).

[15] M.F.L. De Volder, S.H. Tawfick, R.H. Baughman, a J. Hart, Carbon nanotubes: present and future commercial applications., Science. 339 (2013) 535-9.

[16] D.C.B. Alves, F.C. Fonseca, F.D. Brandão, K. Krambrock, A.S. Ferlauto, Temperature dependence of the electrical properties of hydrogen titanate nanotubes, J. Appl. Phys. 116 (2014) 184307.

[17] C. Zhi, Y. Bando, C. Tang, D. Golberg, Boron nitride nanotubes, Mater. Sci. Eng. R Reports. 70 (2010) $92-111$.

[18] I. Heller, W.T.T. Small, S.G. Lemay, C. Dekker, Probing macrophage activity with carbon-nanotube sensors, Small. 5 (2009) 2528-2532. 
[19] G. Ciofani, V. Raffa, A. Menciassi, A. Cuschieri, Boron nitride nanotubes: An innovative tool for nanomedicine, In Vitro. (2009) 8-10.

[20] G. Ciofani, S. Danti, G.G. Genchi, B. Mazzolai, V. Mattoli, Boron Nitride Nanotubes : Biocompatibility and Potential Spill-Over in Nanomedicine, (2013) 1-14.

[21] T.H. Ferreira, A. Marino, A. Rocca, I. Liakos, S. Nitti, A. Athanassiou, et al., Folate-grafted boron nitride nanotubes: Possible exploitation in cancer therapy, Int. J. Pharm. 481 (2015) 56-63.

[22] P.W. Barone, S. Baik, D. a Heller, M.S. Strano, Near-infrared optical sensors based on single-walled carbon nanotubes., Nat. Mater. 4 (2005) 86-92.

[23] E.S. Jeng, A.E. Moll, A.C. Roy, J.B. Gastala, M.S. Strano, Detection of DNA hybridization using the nearinfrared band-gap fluorescence of single-walled carbon nanotubes, Nano Lett. 6 (2006) 371-375.

[24] T.H. Ferreira, P.R.O. Silva, R.G. Santos, E.M.B. Sousa., A Novel Synthesis Route to Produce Boron Nitride Nanotubes for Bioapplications, J. Biomater. Nanobiotechnol. 02 (2011) 426-434.

[25] M. Ishiyama, H. Tominaga, M. Shiga, K. Sasamoto, Y. Ohkura, K. Ueno, A combined assay of cell viability and in vitro cytotoxicity with a highly water-soluble tetrazolium salt, neutral red and crystal violet., Biol. Pharm. Bull. 19 (1996) 1518-20.

[26] M. Zheng, Y. Liu, Y. Gu, Z. Xu, Synthesis and characterization of boron nitride sponges as a novel support for metal nanoparticles, Sci. China Ser. B Chem. 51 (2008) 205-210.

[27] T. Elzein, M. Nasser-Eddine, C. Delaite, S. Bistac, P. Dumas, FTIR study of polycaprolactone chain organization at interfaces, J. Colloid Interface Sci. 273 (2004) 381-387.

[28] K. Phillipson, J.N. Hay, M.J. Jenkins, Thermal analysis FTIR spectroscopy of poly( $\varepsilon$-caprolactone), Thermochim. Acta. 595 (2014) 74-82.

[29] S. Agarwal, C. Speyerer, Degradable blends of semi-crystalline and amorphous branched poly(caprolactone): Effect of microstructure on blend properties, Polymer (Guildf). 51 (2010) 1024-1032.

[30] M.G. McKee, G.L. Wilkes, R.H. Colby, T.E. Long, Correlations of Solution Rheology with Electrospun Fiber Formation of Linear and Branched Polyesters, Macromolecules. 37 (2004) 1760-1767.

[31] M. Terrones, H. Terrones, Y. Bando, D. Golberg, Pure and doped boron nitride nanotubes More than ten years ago, it was suggested theoretically that boron, Rev. Lit. Arts Am. 10 (2007) 30-38.

[32] H. Wang, Y. Wang, Y. Yang, X. Li, C. Wang, Photoluminescence properties of the rare-earth ions in the TiO2 host nanofibers prepared via electrospinning, Mater. Res. Bull. 44 (2009) 408-414.

[33] H. Kweon, M.K. Yoo, I.K. Park, T.H. Kim, H.C. Lee, H.-S. Lee, et al., A novel degradable polycaprolactone networks for tissue engineering., Biomaterials. 24 (2003) 801-8.

[34] S. Sharma, S. Mohanty, D. Gupta, M. Jassal, A.K. Agrawal, R. Tandon, Cellular response of limbal epithelial cells on electrospun poly- $\varepsilon$-caprolactone nanofibrous scaffolds for ocular surface bioengineering: a preliminary in vitro study., Mol. Vis. 17 (2011) 2898-910.

[35] Y.M. Ju, J.S. Choi, A. Atala, J.J. Yoo, S.J. Lee, Bilayered scaffold for engineering cellularized blood vessels., Biomaterials. 31 (2010) 4313-21.

[36] E.S. Jeng, J.D. Nelson, K.L.J. Prather, M.S. Strano, Detection of a single nucleotide polymorphism using single-walled carbon-nanotube near-infrared fluorescence, Small. 6 (2010) 40-43.

Citation: T. Ferreira et al., "Physicochemical Characterization and Cell Adhesion Evaluation of Poly- $\varepsilon-$ Caprolactone/BNNT Nanofibers Produced through Electrospinning Process", International Journal of Advanced Research in Chemical Science (IJARCS), vol. 4, no. 10, pp. 35-43, 2017. http://dx.doi.org/ 10.20431/ 2349-0403.0410004

Copyright: (c) 2017 Authors. This is an open-access article distributed under the terms of the Creative Commons Attribution License, which permits unrestricted use, distribution, and reproduction in any medium, provided the original author and source are credited. 